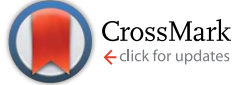

Cite this: J. Mater. Chem. A, 2016, 4, 5032

Received 22nd January 2016 Accepted 2nd March 2016

DOI: $10.1039 / c 6 t a 00391 e$

www.rsc.org/MaterialsA

\section{Roll to roll compatible fabrication of inverted organic solar cells with a self-organized charge selective cathode interfacial layer $\dagger$}

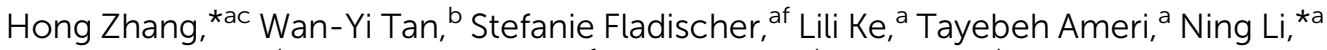 \\ Mathieu Turbiez, ${ }^{d}$ Erdmann Spiecker, ${ }^{f}$ Xu-Hui Zhu, ${ }^{\text {tb }}$ Yong Cao $^{\mathrm{b}}$ \\ and Christoph J. Brabec*ae
}

We successfully demonstrate a simple approach to printing efficient, inverted organic solar cells (OSCs) with a self-organized charge selective cathode interface layer based on the small-molecule Phen-NaDPO. Different from previous studies, Phen-NaDPO molecules were blended into a polymer/fullerene blend, comprising a low bandgap diketopyrrolopyrrole-quinquethiophene alternating copolymer pDPP5T-2 and phenyl- $\mathrm{C}_{61}$-butyric acid methyl ester $\left(\mathrm{PC}_{61} \mathrm{BM}\right)$, and processed by doctor blading in air. We observed a spontaneous, surface energy driven migration of Phen-NaDPO towards the $\mathrm{ZnO}$ interface and a subsequent formation of electron selective and barrier free extraction contacts. In the presence of $0.5 \mathrm{wt} \%$ Phen-NaDPO, a PCE of $5.4 \%$ was achieved for the inverted device based on an ITO/ZnO cathode. Notably, the photovoltaic performances remained at the same level with increasing the Phen-NaDPO concentration in the active layer from 0.25 to $1 \mathrm{wt} \%$. Furthermore, this approach could be proven to effectively work with other cathodes such as bare ITO and ITO/AZO. The self-organization of Phen-NaDPO through spontaneous vertical phase separation is mainly attributed to its high surface energy and strong interaction with the cathode material. The present results highlight that a self-organized cathode interfacial material processed from a "ternary" active layer is fully compatible with the requirements for roll-to-roll fabrication of inverted organic solar cells.

${ }^{a}$ Institute of Materials for Electronics and Energy Technology (i-MEET), Friedrich-Alexander-University Erlangen-Nuremberg, Martensstraße 7, 91058 Erlangen, Germany.E-mail: hong.zhang@fau.de; ning.li@fau.de; christoph.brabec@ fau.de; Fax: +49-9131-8528495; Tel: +49-9131-8527722

${ }^{b}$ State Key Laboratory of Luminescent Materials and Devices (SKLLMD), Institute of Polymer Optoelectronic Materials and Devices, South China University of Technology (SCUT), 510640 Guangzhou, China. E-mail: xuhuizhu@scut.edu.cn

${ }^{c}$ Erlangen Graduate School in Advanced Optical Technologies (SAOT), Paul-GordanStraße 6, 91052 Erlangen, Germany

${ }^{d} B A S F$ Schweiz AG, Schwarzwaldallee 215, CH-4002 Basel, Switzerland

${ }^{e}$ Bavarian Center for Applied Energy Research (ZAE Bayern), Haberstraße 2a, 91058, Erlangen, Germany

${ }^{f}$ Center for Nanoanalysis and Electron Microscopy (CENEM), Friedrich-AlexanderUniversity Erlangen-Nuremberg, Cauerstrasse 6, 91058 Germany

$\dagger$ Electronic supplementary information (ESI) available. See DOI: 10.1039/c6ta00391e

\section{Introduction}

Organic photovoltaics (OPVs) have attracted significant academic and industrial interest due to their exceptional microstructure in combination with unique advantages such as being light-weight, flexible, transparent, and rich in color and potentially being a low-cost and easy to manufacture technology via roll-to-roll processing. ${ }^{1-5}$ In this context, it is critically important to develop materials and device technologies that meet with the requirements for large-area wet processing. Over the past decade, various approaches, including the synthesis of novel materials, customized device architectures, sophisticated interface engineering and advanced processing techniques were employed to continuously improve the power conversion efficiencies (PCEs) of OPV devices. ${ }^{6-9}$ Highly efficient OPV devices with record efficiencies above $10 \%$ were manufactured by spincoating under inert conditions. ${ }^{\mathbf{1 0 - 1 2}}$ However, spin-coating is a batch process and as such incompatible with the visionary concept of fully printed or coated organic solar modules.

One of the relevant problems in the printed OPV technology is the formation of a selective and barrier free extraction contact at the cathode. ${ }^{\mathbf{8} 13}$ The most popular electron extraction layers (EELs) reported so far are based on self-assembled monolayers (SAMs), ${ }^{14}$ conjugated and non-conjugated polymers ${ }^{15,16}$ or alkali metal complexes, ${ }^{8}$ which provide selective electron extraction in parallel to sufficient hole blocking functionality. However, most of these EELs are sequentially processed from solution, along with other functional layers, and are fairly thickness-sensitive. ${ }^{8,15}$ In this context, this thickness-sensitivity is further watched with concern as it may restrict large-area high-speed roll-to-roll processing, and vulgarizes the necessity to further explore novel interfacial materials with improved optical, electrical and thermodynamic properties. ${ }^{17}$

It is worth noting that roll-to-roll compatible processed solar cells usually show a quite remarkable drop in efficiencies compared to spin-coated devices, highlighting the importance of the development of a stable, reliable production process which should allow a minimization of the performance 
losses. ${ }^{18,19}$ Selecting appropriate materials for the printing process is a crucial problem to be considered, since all the layers of organic solar cells are supposed to deposit from solutions and inevitably interact with each other. To focus this study on the development of selective and barrier free interfacial layers, a diketopyrrolopyrrole-quinquethiophene alternating copolymer, pDPP5T-2, is used as the electron donor owing to its excellent air-stability and reliability for up-scaling fabrication of solar cells along with promising photovoltaic performance. ${ }^{\mathbf{1 9 , 2 0}}$ Organic solar modules based on pDPP5T-2 have been recently demonstrated on a large area of $64 \mathrm{~cm}^{2}$ by connecting 16 single cells in series. ${ }^{21}$ The semi-transparent solar modules with all the layers printed in air reached a fully open circuit voltage of $9.10 \mathrm{~V}$ and a module efficiency of $2.34 \%$. Recently, several groups successfully demonstrated the formation of EELs via spontaneous vertical phase separation of small or high surface energy additives in organic $\mathrm{BHJ}$ composite wet films and subsequent self-organization at the cathode interface during drying. ${ }^{22-27}$ Depending on the surface energy and the substrate, these layers may migrate to the bottom or top interface of the active layer, thus forming conventional (bottom-anode) or inverted (bottomcathode) architecture. Wang et al. ${ }^{22}$ demonstrated that aminobased cathode interfacial materials (fullerene [6,6]-phenyl- $\mathrm{C}_{61^{-}}{ }^{-}$ butyric acid 2-((2-(dimethylamino)-ethyl)(methyl)amino)ethyl ester (PCBDAN) and poly[(9,9-bis(3-( $N, N$-dimethylamino)propyl)2,7-fluorene)-alt-2,7-(9,9-dioctylfluorene)] (PFN)) improve electron selective contacts with ITO when added into the P3HT:PC ${ }_{61} \mathrm{BM}$ solution at small concentrations. Lee et al. ${ }^{23}$ showed that polyethyleneimine (PEI) aligned on top of PEDOT:PSS when mixed into the photoactive materials, thereby significantly simplifying the multi-junction processing. In this approach PEI had to be dissolved in a polar solvent, such as 2-methoxyethanol, before mixing with the photoactive solutions. Addition of the poor (polar) solvents to the photovoltaic semiconductor ink may complicate the microstructure formation of the active layer..$^{5,28,29}$

The investigations on self-organized EELs reported so far were exclusively achieved by spin-coating a "ternary" blend of the $\mathrm{BHJ}$ photoactive materials with the EEL as an additive under inert atmosphere conditions. This significantly hampers the relevance of these findings for the scenario of the roll to roll processing line. ${ }^{22-24}$

In this study we demonstrate that self-organization of EELs is indeed compatible to roll to roll processing under ambient conditions. A small molecule (2-(1,10-phenanthrolin-3-yl) naphth-6-yl)diphenylphosphine oxide (Phen-NaDPO Fig. 1a) is shown to form a selective electron layer onto various metal oxide surfaces via vertical phase separation from a "ternary" $\mathrm{BHJ}$ photoactive solution doctor-bladed in air. As a versatile cathode modification material, Phen-NaDPO possesses multiple attractive attributes, combining facile synthesis and purification, a high glass transition temperature $\left(T_{\mathrm{g}} \approx 116^{\circ} \mathrm{C}\right)$ and electron mobility $\left(\mu_{\mathrm{e}}=\sim 10^{-4}\right.$ to $10^{-3} \mathrm{~cm}^{2} \mathrm{~V}^{-1} \mathrm{~s}^{-1}$ @8 $\left.\times 10^{5} \mathrm{~V} \mathrm{~cm}^{-1}\right)$ with a low-lying HOMO level of $-6.1 \mathrm{eV}$ and LUMO level of $-2.74 \mathrm{eV} .^{\mathbf{3 0 , 3 1}}$ Furthermore, it should be emphasized, different from the strongly hydrophilic CIMs such as PEI, Phen-NaDPO exhibits good solubility in weakly polar solvents, such as chlorobenzene (CB) and $o$-dichlorobenzene (ODCB). Consequently, no additional polar solvent is needed in processing the active layer, thus facilitating the large-scale roll-to-roll production with high reproducibility and reliability.

\section{Results and discussion}

The photoactive materials studied in this work are based on a low bandgap diketopyrrolopyrrole-quinquethiophene alternating copolymer, pDPP5T-2, as the electron donor and phenyl$\mathrm{C}_{61}$-butyric acid methyl ester $\left(\mathrm{PC}_{61} \mathrm{BM}\right)$ as the electron acceptor (Fig. 1a). The inverted organic solar cells (ITO/ZnO/pDPP5T2:PC ${ }_{61} \mathrm{BM}:$ Phen-NaDPO/ $\mathrm{MoO}_{3} / \mathrm{Ag}$ ) involving a small amount of Phen-NaDPO as the additive and (ITO/ZnO/Phen-NaDPO/ pDPP5T-2:PCBM/ $\mathrm{MoO}_{3} / \mathrm{Ag}$ ) with Phen-NaDPO as a thin interlayer are shown in Fig. 1b, respectively. The relevant energy levels are illustrated in Fig. 1c. The photoactive layer as well as the zinc oxide layer was fabricated by doctor blading under ambient conditions.

Introducing a small amount of Phen-NaDPO into the pDPP5T2:PCBM blend results in considerably increased PCEs as compared to the reference device without the EEL (Fig. 2 and Table 1). For instance, the reference device without the EEL exhibits a PCE of $4.0 \%$ with an open circuit voltage $\left(V_{\mathrm{OC}}\right)$ of $0.56 \mathrm{~V}$, a short circuit current density $\left(J_{\mathrm{SC}}\right)$ of $12.12 \mathrm{~mA} \mathrm{~cm}^{-2}$ and a fill factor (FF) of $59.59 \%$. By adding $0.5 \mathrm{wt} \%$ Phen-NaDPO into the semiconductor ink, $J_{\mathrm{SC}}$ and $\mathrm{FF}$ are increased to $13.51 \mathrm{~mA}$ $\mathrm{cm}^{-2}$ and $69.06 \%$, respectively, yielding a PCE of $5.22 \%$ while $V_{\text {OC }}$ remains unaffected. Upon further increasing the Phen-NaDPO concentration to $2 \mathrm{wt} \% J_{\mathrm{SC}}$ continues to rise, but the FF starts to drop more rapidly, resulting in an overall lower PCE of $4.79 \%$.

The external quantum efficiency (EQE) spectra of the solar cells with $0-2 \mathrm{wt} \%$ Phen-NaDPO are presented in Fig. 2b. The photocurrents calculated by integrating the EQE spectra with the AM1.5 solar spectrum reproduce the trend observed from $j-V$ analysis and are basically consistent with the experimental values summarized in Table 1 . The EQE spectra reveal that incorporating a small amount of Phen-NaDPO as an additive in the blend dominantly enhances the response in the short wavelength regime (350-450 nm), i.e. where PCBM absorbs, as well as the long wavelength regime $(600-800 \mathrm{~nm})$. It is noteworthy that the identical trend was observed for the otherwise identical solar cells with $\mathrm{Ba}(\mathrm{OH})_{2}$ as the EEL. ${ }^{8}$ The analogue with $\mathrm{Ba}(\mathrm{OH})_{2}$ suggests a similar functionality of Phen-NaDPO, namely a more efficient electrode extraction resulting in reduced $2^{\text {nd }}$ order recombination at the interface. We note that there may be an effectively self-organized Phen-NaDPO layer on the $\mathrm{ZnO}$ substrate.

In order to gain a clear evidence for the observed self-organization, solar cells with the architecture ITO/ZnO/PhenNaDPO/pDPP5T-2:PCBM/ $\mathrm{MoO}_{3} / \mathrm{Ag}$ were fabricated, where Phen-NaDPO solution was separately bladed as the EEL atop ZnO. The current density-voltage $(j-V)$ characteristics of the inverted OSCs with different thick Phen-NaDPO layers are summarized in Fig. S1 and Table S1 (ESI). $\dagger$ The best PCE of $4.80 \%$ was achieved at a blading speed of $10 \mathrm{~mm} \mathrm{~s}^{-1}$ with a $V_{\mathrm{OC}}$ of $0.56 \mathrm{~V}, J_{\mathrm{SC}}$ of $13.94 \mathrm{~mA} \mathrm{~cm}^{-2}$ and $\mathrm{FF}$ of $61.6 \%$. The interlayer 

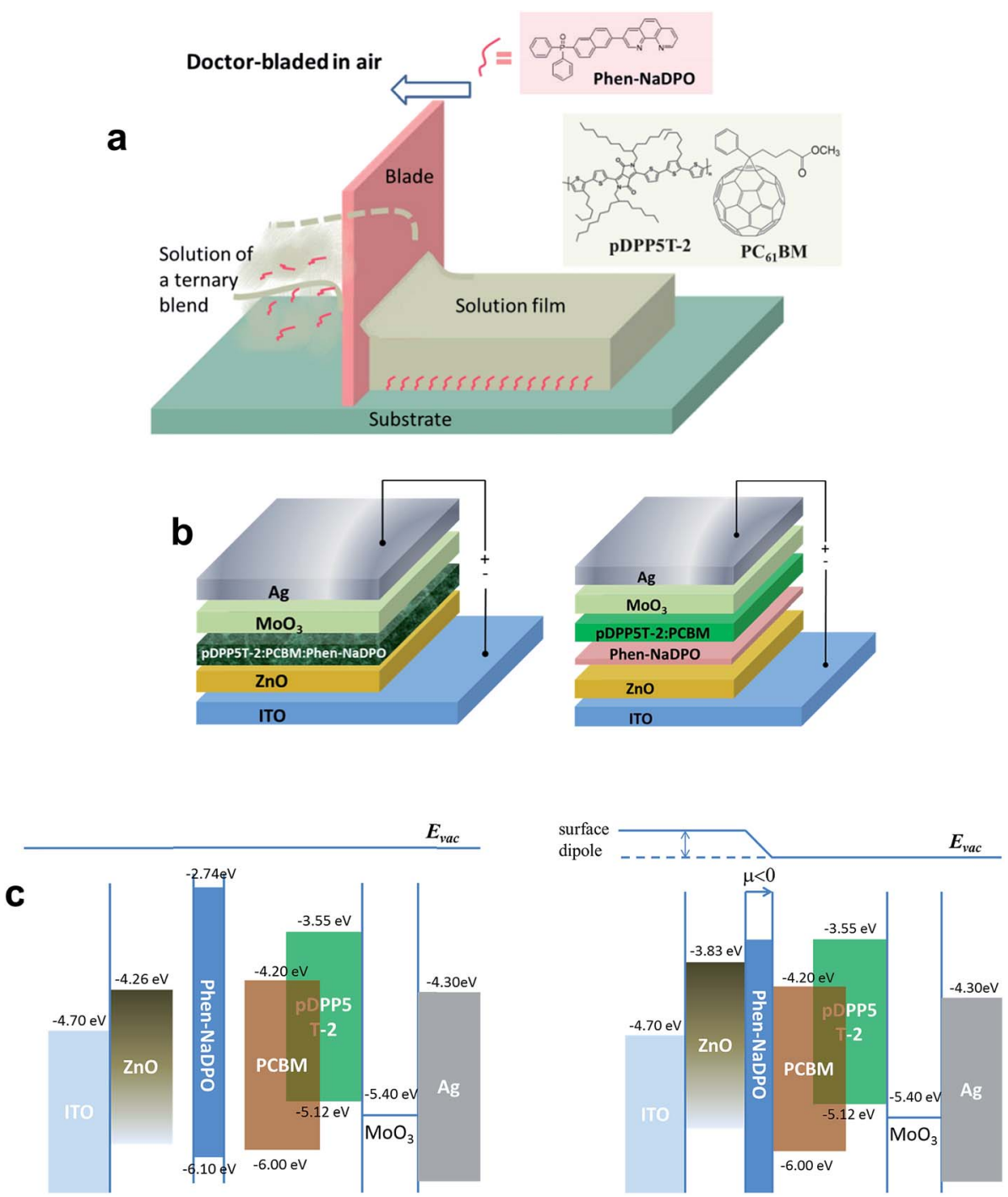

Fig. 1 (a) Conceptual diagram for the active layer deposition with a self-organizing EEL by doctor-blading as well as the chemical structures of pDPP5T-2, PC 61 BM and Phen-NaDPO. (b) Schematic illustration of the device configuration of the OSCs with Phen-NaDPO as an additive in the active layer (left) and as an interlayer between the active layer and ZnO (right). The donor (pDPP5T-2) to acceptor (PCBM) ratio was kept to 1 : 1.5 by weight fraction. The active layer thickness was optimized to $\sim 120 \mathrm{~nm}$. (c) Schematic energy level diagram of the functional materials before contact formation (left) and after contact formation (right). After contact formation, the interaction of Phen-NaDPO with the ZnO substrate resulted in lowering the vacuum level of the cathode.

thickness was ca. $8 \mathrm{~nm}$, subject to possible variation after doctor blading the active layer. Doctor blading methanol for reference purposes appeared to have no significant influence on the PCE. These results show that self-organization from a ternary formulation indeed is a plausible scenario as it leads to generally better PCEs as a dedicated Phen-NaDPO layer on top of ZnO.

Phen-NaDPO was already shown to be a versatile cathode modification layer for Ag, ITO and HOPG due to its strong interaction with the substrates. ${ }^{30}$ We studied this effect for various metal oxide substrates using Kelvin probe spectroscopy (see ESI Table S2 $\dagger$ ). As expected, coating a thin layer of PhenNaDPO decreases the work functions for ITO, ITO/AZO and ITO/ $\mathrm{ZnO}$ to $4.01,3.92$ and $3.83 \mathrm{eV}$, respectively. Bare ITO cathodes strongly lack selectivity and are thus more prone to recombination losses as compared to ITO/ZnO or ITO/AZO. We therefore varied the Phen-NaDPO layer thickness for ITO/PhenNaDPO/pDPP5T-2:PCBM/ $\mathrm{MoO}_{3} / \mathrm{Ag}$ devices as a function of work function (see ESI Fig. S2 $\dagger$ ). With increasing Phen-NaDPO layer thickness the work function decreases from 4.80 to $4.01 \mathrm{eV}$ before leveling out around $4.17 \mathrm{eV}$. Apparently, $V_{\mathrm{OC}}, J_{\mathrm{SC}}$ and FF follow the trend of the work function, underpinning the formation of a more and more charge selective interface. Previous reports suggested that Phen-NaDPO may even participate in the charge transfer process from PCBM to the electrode, a hypothesis which minimizes the energy loss during electron extraction. ${ }^{30}$

Finally, we found that self-organization from "ternary" composites works well for bare ITO and ITO/AZO substrates (see ESI Fig. S3 and S4†). However, we note that the modified ITO electrodes are constantly performing inferior to the 

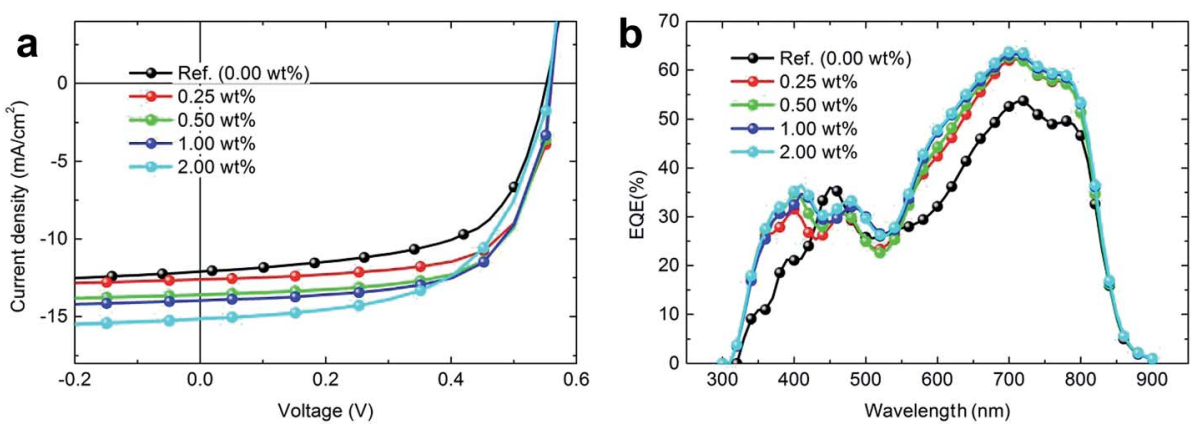

Fig. 2 (a) $j-V$ characteristics of inverted OSCs (ITO/ZnO/"ternary" active layer/ $\mathrm{MoO}_{3} / \mathrm{Ag}$ ) with different Phen-NaDPO contents under simulated AM1.5 illumination (100 $\mathrm{mW} \mathrm{cm}^{-2}$ ). (b) EQE spectra of inverted OSCs corresponding to (a).

Table 1 Summary of the $j-V$ characteristics of inverted OSCs (ITO/ZnO/"ternary" active layer/MoO $3 / \mathrm{Ag}$ ) with varying Phen-NaDPO contents under simulated AM1.5 illumination $\left(100 \mathrm{~mW} \mathrm{~cm}^{-2}\right)^{a}$

\begin{tabular}{lllll}
\hline Phen-NaDPO $(\mathrm{wt} \%)$ & $V_{\mathrm{OC}}(\mathrm{V})$ & $J_{\mathrm{SC}}\left(\mathrm{mA} \mathrm{cm}{ }^{-2}\right)$ & FF $(\%)$ & PCE $(\%)$ avg $(\mathrm{best})$ \\
\hline 0 & 0.56 & $-12.12 \pm 0.56$ & $59.59 \pm 0.50$ & $4.00(4.30)$ \\
0.25 & 0.56 & $-13.06 \pm 1.01$ & $70.04 \pm 0.56$ & $5.11(5.46)$ \\
0.50 & 0.56 & $-13.51 \pm 0.30$ & $6.06 \pm 0.98$ & $5.22(5.40)$ \\
1.00 & 0.56 & $-13.83 \pm 0.26$ & $58.85 \pm 0.85$ & $5.10(5.17)$ \\
2.00 & 0.56 & $-14.63 \pm 0.76$ & 5.00 & $4.79(4.98)$
\end{tabular}

${ }^{a}$ Each value represents the average from five cells corresponding to different positions on one substrate.

analogous ITO/ZnO or ITO/AZO cathodes, mainly because they are failing to deliver full $V_{\mathrm{OC}}$. We speculate that incomplete electrode coverage may cause $V_{\mathrm{OC}}$ losses via surface recombination at the electrode. Recently we showed that the ITO/AZO cathodes are sufficiently modified by inserting a barium hydroxide layer between the AZO and the active layer. ${ }^{8}$ As a result, the performance of pDPP5T-2 based OSCs was significantly improved from $3.3 \%$ to $6 \%$. In Table $\mathrm{S} 3, \uparrow$ the $j-V$ characteristics of the "ternary" blend active layer devices based on AZO are compared with those of the devices with bilayer $\mathrm{AZO} / \mathrm{Ba}(\mathrm{OH})_{2}$ as the EEL. We can find that the performance of the optimized "ternary" blend active layer devices is comparable with those of the devices with bilayer $\mathrm{AZO} / \mathrm{Ba}(\mathrm{OH})_{2}$ as the EEL, suggesting a one-step "ternary" blend solution process to obtain an ideal EEL/active layer with a simplified one-layer structure to overcome a contact barrier between the n-type metal oxides and the lowest unoccupied molecular orbital (LUMO) of the acceptor of the active layer. This simple one-step solution processing leading to a de facto bilayer with a bottom cathode interfacial layer and a top $\mathrm{BHJ}$ photoactive layer is advantageous for high-volume roll-to-roll printing to effectively reduce the production cost and time.

To directly evidence the distribution of Phen-NaDPO at the substrate, we investigated the device cross-section by transmission electron microscopy (TEM). Energy dispersive X-ray spectroscopy (EDXS) in the scanning transmission electron microscopy (STEM) mode reveals the composition of the single layers and interfaces in OSCs. In this case the phosphorus signal of Phen-NaDPO should be used to determine the material distribution as this element is not present in the pDPP5T2:PCBM blend. STEM EDXS analyses have been performed at the $\mathrm{ZnO} /$ active layer interface of the device with a small amount ( $\sim 2$ wt $\%)$ of Phen-NaDPO in the pDPP5T-2:PCBM blend, showing the elemental composition at different locations of the cross-section (see Fig. S5†). The elemental mappings of zinc, phosphorus, carbon, oxygen, silicon and sulfur are shown in Fig. S5b. $\uparrow$ Note that the small amount of $\mathrm{P}$ is observed around the $\mathrm{ZnO}$ interface rather than in the "ternary" blend composite. However, considering the phosphoric acid ligands of $\mathrm{ZnO}$ from NanoGrade, we further investigated the device with a small amount ( $\sim 2 \mathrm{wt} \%$ ) of Phen-NaDPO in the pDPP5T-2:PCBM blend at the bare ITO substrate (see Fig. S6 $\dagger$ ). In this case no P signal was detected by EDXS, concluding that the amount of $\mathrm{P}$ in the "ternary" blend composite is below the detection limit of this EDXS system. Therefore we decided to indirectly prove the accumulation of Phen-NaDPO at the substrate and analyzed the surface energy at the top and bottom surfaces of the semiconductor.

Self-organization is influenced by the blend composition, substrate and surface energy. ${ }^{22,32-35}$ The surface energies of ITO, pDPP5T-2, PCBM, Phen-NaDPO, pDPP5T-2:PCBM and pDPP5T2:PCBM:Phen-NaDPO were determined by measuring the droplet's contact angle for three liquids (ultrapure water, diiodomethane and ethylene glycol) using the Owens-Wendt and Kaelble method (Fig. 3a). ${ }^{36}$ The ITO and Phen-NaDPO surfaces exhibit rather high surface energies $\left(\gamma_{\text {гто }}=62.32 \mathrm{mN} \mathrm{m}^{-1}\right.$ and $\gamma_{\text {Phen-NaDPo }}=49.81 \mathrm{mN} \mathrm{m}^{-1}$ ) originating from their terminal hydroxyls, phosphine oxide and phenanthroline moieties. In 

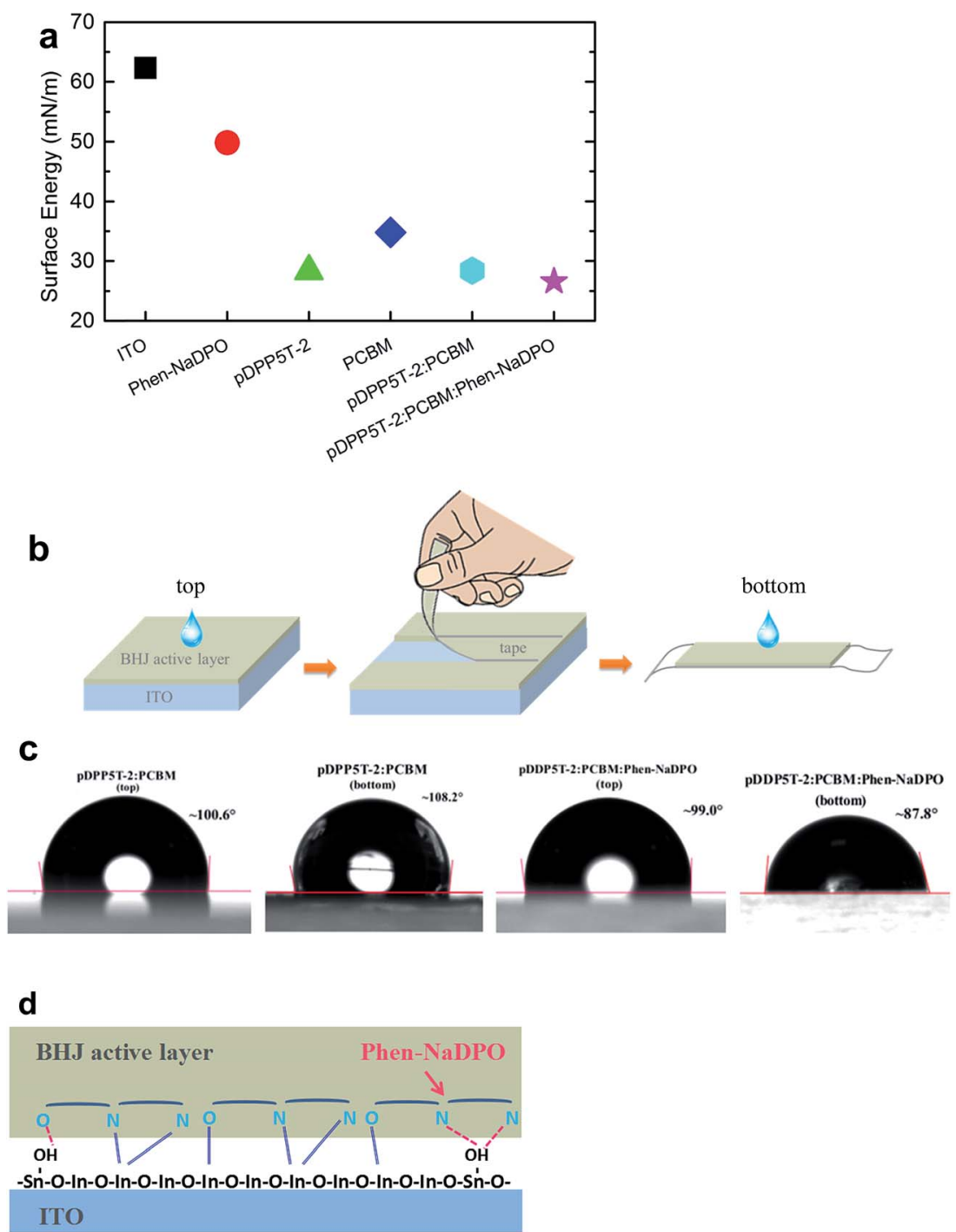

Fig. 3 (a) The calculated surface energies of materials studied in this work. (b) A schematic of the peeling process of the "ternary" active layer blend film to expose the front contact. (c) Photographs of water droplets on the front/back substrate surfaces coated with films of "binary" and "ternary" active layer blend film. (d) Illustration of the self-organized molecules of Phen-NaDPO at the ITO interface, which show potential interactions with the surface metal ions and the residual hydroxyl groups.

contrast, pristine pDPP5T-2 and PCBM show surface energies of 28.34 and $34.78 \mathrm{mN} \mathrm{m}^{-1}$, respectively. The surface energies of the composite films pDPP5T-2:PCBM and pDPP5T-2:PCBM:PhenNaDPO are found to be 28.37 and $26.61 \mathrm{mN} \mathrm{m}^{-1}$, respectively, which are almost identical to that of the pristine pDPP5T-2. pDPP5T-2 and PCBM exhibit low surface energies due to their hydrophobic diketopyrrolopyrrole segment and alkyl side chains. Similar values were reported for other blends previously. ${ }^{22,23,32}$

Next we peeled off the substrate to expose the bottom contact, i.e. the bottom surface of the "ternary" blend film (schematically illustrated in Fig. 3b). As shown in Fig. 3c and $\mathrm{S} 7, \dagger$ the top surface of the "ternary" blend film is of hydrophobic nature with a water contact angle of $\approx 99.0^{\circ}$, similar to the value for the top surface of the binary blend film (water contact angle $\approx 100.6^{\circ}$ ). Pristine ITO and Phen-NaDPO are hydrophilic (water contact angle $\approx 24.1^{\circ}$ and $58.8^{\circ}$ respectively). The bottom surface of the "binary" film is hydrophobic with a contact angle of $\approx 108.2^{\circ}$ which is significantly reduced to $87.8^{\circ}$ upon addition of Phen-NaDPO for the "ternary" blend film. These measurements clearly reveal that the "ternary" system minimizes its free energy by assembling the highsurface energy components (i.e. Phen-NaDPO) at the substrate while the low-surface energy components (i.e. pDPP5T-2 and PCBM) accumulate at the surface. This is further in excellent agreement with the observation that the surface energy of pDPP5T-2:PCBM:Phen-NaDPO is nearly identical to that of pDPP5T-2:PCBM, suggesting that no hydrophilic Phen-NaDPO is existent at the surface of the film.

Fig. 3d illustrates the self-organized molecules of Phen-NaDPO at the ITO interface, which show potential interactions with the surface metal ions and the residual hydroxyl groups, leading to reduction of the work function of the ITO substrate. Furthermore, the low HOMO of Phen-NaDPO (Fig. 1) may reduce the electron-hole recombination at the cathode. 
Finally, Fig. S8† shows atomic force microscopy (AFM) images for various "ternary" Phen-NaDPO/BHJ composites. For binary BHJ composites, a smooth surface with a root mean square (RMS) roughness value around $2.15 \mathrm{~nm}$ is noted. Small changes in the surface topography are denoted upon addition of Phen-NaDPO. The surface roughness increases from 2.25, 2.49, and 2.64 to $3.02 \mathrm{~nm}$ with increasing amount of Phen-NaDPO in the active layer from $0.25,0.5$, and 1 to $2 \mathrm{wt} \%$. The RMS roughness of $\mathrm{ZnO}$ is determined with $3.90 \mathrm{~nm}$ and appears unchanged after modification with Phen-NaDPO (Fig. S8g $\dagger$ ).

\section{Conclusion}

In conclusion, we have successfully proved that the self-assembly and self-formation of electron-extraction layers from "ternary" blend solutions is compatible with roll to roll processing strategies. A simple approach of fabricating efficient inverted OSCs with a self-organizing small-molecule electron extraction layer Phen-NaDPO by doctor-blading was demonstrated on ITO, ITO/ZnO and ITO/AZO. A PCE of $5.4 \%$ was achieved for the inverted device based on an ITO/ZnO cathode. The photovoltaic performance remained quite stable for a rather wide range of Phen-NaDPO concentrations in the active layer blend, thus offering tremendous prospects for a stable and reliable process development. The self-organization of Phen-NaDPO through vertical phase separation is attributed to its high surface energy and strong interactions with the cathode material. The present study shows that utilizing the self-organization processes of appropriate interfacial materials from "ternary" active layer formulations indeed is a promising strategy towards simplified roll-to-roll processing of organic solar cells.

\section{Experimental section}

\section{Materials}

pDPP5T-2 (batch: GKS1-001) was provided by BASF. PC $_{61} \mathrm{BM}$ (99\%) was purchased from Solenne BV. ZnO nanoparticle suspension solution (Lot\#5039) was provided by NanoGrade. Phen-NaDPO was synthesized in accordance with the literature method. ${ }^{30}$ All materials were used as received.

Synthesis of Al doped zinc oxide (AZO): $\mathrm{Zn}(\mathrm{OAc})_{2} \cdot 2 \mathrm{H}_{2} \mathrm{O}$ $(20 \mathrm{~g}), \mathrm{Al}(\mathrm{OH})(\mathrm{OAc})_{2}(0.3 \mathrm{~g})$ and Zonyl FSO-100 (0.6 g) were mixed in demineralized water $(200 \mathrm{~mL})$. The mixture was stirred for $2 \mathrm{~h}$ and filtered through a 0.45 micrometer filter to remove the insoluble material. The functionality of AZO in inverted OSCs was fully investigated previously. ${ }^{37,38}$

\section{Device processing}

Fabrication of organic solar cells. Thin films of the organic layers as well as the oxide electron-collection layer were fabricated via doctor-blading under ambient conditions with the structures shown in Fig. 1. Pre-structured ITO coated glass substrates were subsequently cleaned in acetone and isopropyl alcohol for $10 \mathrm{~min}$ each. After drying, the substrates were coated with a $50 \mathrm{~nm}$ thick AZO layer or $50 \mathrm{~nm}$ thick ZnO layer via doctor blading, respectively. Conversion of the precursor to AZO via hydrolysis was achieved by heating the samples to $140{ }^{\circ} \mathrm{C}$ for $5 \mathrm{~min}$. The AZO precursor was synthesized as optimized and reported earlier based on zinc acetate and aluminum nitrate in ethanol. The $\mathrm{ZnO}$ layer was annealed on a hot plate at $80{ }^{\circ} \mathrm{C}$ for $5 \mathrm{~min}$. Subsequently, a $120 \mathrm{~nm}$ thick thin film based on the "ternary" solution pDPP5T-2:PC ${ }_{61}$ BM:Phen-NaDPO was deposited on top of the AZO or $\mathrm{ZnO}$ layer. The blend pDPP5T-2 : $\mathrm{PC}_{61} \mathrm{BM}(1: 2)$ was prepared by dissolving in a mixed solvent of $90 \%$ chloroform and $10 \%$ $o$-dichlorobenzene at a concentration of $24 \mathrm{mg} \mathrm{mL}^{-1}$ in total. For device optimization, Phen-NaDPO was added in different weight ratios relative to pDPP5T- 2 and $\mathrm{PC}_{61} \mathrm{BM}$. Finally $10 \mathrm{~nm} \mathrm{MoO}_{3}$ and $100 \mathrm{~nm} \mathrm{Ag}$ were deposited sequentially under $5 \times 10^{-6}$ Torr by thermal evaporation through a shadow mask to form an active area of $10.4 \mathrm{~mm}^{2}$.

In the case of the OSCs based on the binary solution pDPP5T2: $\mathrm{PC}_{61} \mathrm{BM}$, an ethanol solution of Phen-NaDPO was doctorbladed first on $\mathrm{ZnO} / \mathrm{ITO}$ substrates at different velocities. The active layer of pDPP5T-2:PC ${ }_{61} \mathrm{BM}$ was then doctor-bladed onto the Phen-NaDPO film. Doctor-blading the blank methanol solution was carried out for comparison. All the other steps were the same as described for the OSCs involving the "ternary" solution pDPP5T-2:PC ${ }_{61}$ BM:Phen-NaDPO.

\section{Characterization methods}

The current-voltage characteristics were measured using a source measurement unit from BoTest through a mask with an opening of $10.4 \mathrm{~mm}^{2}$. Illumination was provided by a solar simulator (Oriel Sol 1A, from Newport) with AM1.5G spectra at $100 \mathrm{~mW} \mathrm{~cm}{ }^{-2}$. External quantum efficiencies were measured by an Enli Technology (Taiwan) EQE measurement system. The morphology was investigated by AFM (Veeco Model D3100, tapping mode). The work function of various substrates w or w/o Phen-NaDPO was measured by using a Kelvin probe (Kelvin Probe Technologies Inc., SKP5050) in air. The thicknesses of the films were measured by a profilometer (Tencor Alpha Step). All devices were tested in ambient air. Cross-sections of the solar cells were prepared for TEM investigations using the in situ liftout technique in a focused ion beam (FIB) instrument, an a FEI Helios NanoLab 660 DualBeam system. TEM investigations were performed on an aberration-corrected FEI Titan Themis $^{3} 300$ TEM with a high brightness field emission gun (X-FEG) operated at $200 \mathrm{kV}$ equipped with a Super-X detector for energy-dispersive $\mathrm{X}$-ray spectroscopy (EDXS). The contact angle and the surface of the bladed and annealed films of pristine materials and their binary and "ternary" blends were measured using a contact angle instrument from Dataphysics (model OCA20). With the values of the initial contact angles, surface energies were calculated using the SCA20-U software and the Owens-Wendt and Kaelble method. The blend films were peeled by strongly adhering the Kapton tape and peeling the films off by hand.

\section{Acknowledgements}

The authors gratefully acknowledge the support of the Cluster of Excellence "Engineering of Advanced Materials (EAM)", Energy Campus Nuremberg (EnCN, Solarfactory), "Synthetic 
Carbon Allotropes" (SFB 953) project, DFG research training group GRK 1896 and the Erlangen Graduate School in Advanced Optical Technologies (SAOT) at the University of ErlangenNuremberg, which is funded by the German Research Foundation (DFG) within the framework of its "Excellence Initiative". This work has been partially funded by the China Scholarship Council (CSC). The authors also thank the support of the Solar Technologies go Hybrid (Sol-Tech) project from the Bavarian Ministry of Science and the Joint Projects of the Helmholtz Institute Erlangen Nürnberg for Renewable Energy Production (HI-ERN) from the Bavarian Ministry of Economic Affairs and Media, Energy and Technology. Nanograde is acknowledged for providing the ZnO nanoparticles suspension. XHZ gratefully acknowledges the support from SCUT, NSF and MOST of China (Grant No. 2014CB643500, 2014ZG0009, 51173051, U1301243, 91333206, 201101C0105067115).

\section{References}

1 C. J. Brabec, N. S. Sariciftci and J. C. Hummelen, Adv. Funct. Mater., 2001, 11, 15-26.

2 C. J. Brabec, S. Gowrisanker, J. J. M. Halls, D. Laird, S. J. Jia and S. P. Williams, Adv. Mater., 2010, 22, 3839-3856.

3 C. J. Brabec, Sol. Energy Mater. Sol. Cells, 2004, 83, 273-292.

4 P. W. M. Blom, V. D. Mihailetchi, L. J. A. Koster and D. E. Markov, Adv. Mater., 2007, 19, 1551-1566.

5 S. Gunes, H. Neugebauer and N. S. Sariciftci, Chem. Rev., 2007, 107, 1324-1338.

6 Y. Y. Liang, Z. Xu, J. B. Xia, S. T. Tsai, Y. Wu, G. Li, C. Ray and L. P. Yu, Adv. Mater., 2010, 22, E135-E138.

7 T. Ameri, N. Li and C. J. Brabec, Energy Environ. Sci., 2013, 6, 2390-2413.

8 H. Zhang, T. Stubhan, N. Li, M. Turbiez, G. J. Matt, T. Ameri and C. J. Brabec, J. Mater. Chem. A, 2014, 2, 18917-18923.

9 G. Li, V. Shrotriya, J. S. Huang, Y. Yao, T. Moriarty, K. Emery and Y. Yang, Nat. Mater., 2005, 4, 864-868.

10 M. A. Green, K. Emery, Y. Hishikawa, W. Warta and E. D. Dunlop, Prog. Photovoltaics, 2015, 23, 805-812.

11 J. B. You, L. T. Dou, K. Yoshimura, T. Kato, K. Ohya, T. Moriarty, K. Emery, C. C. Chen, J. Gao, G. Li and Y. Yang, Nat. Commun., 2013, 4, 1446.

12 J. D. Chen, C. H. Cui, Y. Q. Li, L. Zhou, Q. D. Ou, C. Li, Y. F. Li and J. X. Tang, Adv. Mater., 2015, 27, 1035-1041.

13 R. Steim, F. R. Kogler and C. J. Brabec, J. Mater. Chem., 2010, 20, 2499-2512.

14 H. L. Yip, S. K. Hau, N. S. Baek, H. Ma and A. K. Y. Jen, Adv. Mater., 2008, 20, 2376-2382.

15 Z. C. He, C. M. Zhong, S. J. Su, M. Xu, H. B. Wu and Y. Cao, Nat. Photonics, 2012, 6, 591-595.

16 Y. H. Zhou, C. Fuentes-Hernandez, J. Shim, J. Meyer, A. J. Giordano, H. Li, P. Winget, T. Papadopoulos, H. Cheun, J. Kim, M. Fenoll, A. Dindar, W. Haske, E. Najafabadi, T. M. Khan, H. Sojoudi, S. Barlow, S. Graham, J. L. Bredas, S. R. Marder, A. Kahn and B. Kippelen, Science, 2012, 336, 327-332.

17 F. C. Krebs, S. A. Gevorgyan and J. Alstrup, J. Mater. Chem., 2009, 19, 5442-5451.
18 M. Hösel, D. Angmo, R. R. Søndergaard, G. A. dos Reis Benatto, J. E. Carlé, M. Jørgensen and F. C. Krebs, Adv. Sci., 2014, 1, 1400002.

19 L. Lucera, P. Kubis, F. W. Fecher, C. Bronnbauer, M. Turbiez, K. Forberich, T. Ameri, H. J. Egelhaaf and C. J. Brabec, Energy Technol., 2015, 3, 373-384.

20 S. Arumugam, D. Cortizo-Lacalle, S. Rossbauer, S. Hunter, A. L. Kanibolotsky, A. R. Inigo, P. A. Lane, T. D. Anthopoulos and P. J. Skabara, ACS Appl. Mater. Interfaces, 2015, 7, 27999-28005.

21 F. Guo, P. Kubis, T. Przybilla, E. Spiecker, A. Hollmann, S. Langner, K. Forberich and C. J. Brabec, Adv. Energy Mater., 2015, 5, 1401779.

22 D. Ma, M. L. Lv, M. Lei, J. Zhu, H. Q. Wang and X. W. Chen, ACS Nano, 2014, 8, 1601-1608.

23 H. Kang, S. Kee, K. Yu, J. Lee, G. Kim, J. Kim, J. R. Kim, J. Kong and K. Lee, Adv. Mater., 2015, 27, 1408-1413.

24 S. C. Chien, F. C. Chen, M. K. Chung and C. S. Hsu, J. Phys. Chem. C, 2012, 116, 1354-1360.

25 D. Qin, P. Cheng, Y. Wang, Y. Fan and X. Zhan, J. Mater. Chem. C, 2015, 4, 1051-1056.

26 X. Li, Y. F. Hu, Z. B. Deng, L. J. Zhu, Y. Wang, D. H. Xu, Y. B. Hou and F. Teng, Phys. Status Solidi A, 2015, 212, 1800-1804.

27 Q. S. Wei, T. Nishizawa, K. Tajima and K. Hashimoto, Adv. Mater., 2008, 20, 2211-2216.

28 F. Machui, S. Langner, X. D. Zhu, S. Abbott and C. J. Brabec, Sol. Energy Mater. Sol. Cells, 2012, 100, 138-146.

29 K. E. Strawhecker, S. K. Kumar, J. F. Douglas and A. Karim, Macromolecules, 2001, 34, 4669-4672.

30 W. Y. Tan, R. Wang, M. Li, G. Liu, P. Chen, X. C. Li, S. M. Lu, H. L. Zhu, Q. M. Peng, X. H. Zhu, W. Chen, W. C. H. Choy, F. Li, J. B. Peng and Y. Cao, Adv. Funct. Mater., 2014, 24, 6540-6547.

31 W. Y. Tan, X. H. Zhu, J. W. Chen and Y. Cao, Conjugated Polymer and Organic Molecules as Cathode Interfacial Materials for Polymer Solar Cells, in Handbook of Polymer and Hybrid Photovoltaics, ed. S. A. Chen, Wiley, submitted on July 31st, 2015, ch. 13.

32 K. Yao, L. Chen, X. Chen and Y. W. Chen, Chem. Mater., 2013, 25, 897-904.

33 T. Ameri, P. Khoram, T. Heumuller, D. Baran, F. Machui, A. Troeger, V. Sgobba, D. M. Guldi, M. Halik, S. Rathgeber, U. Scherf and C. J. Brabec, J. Mater. Chem. A, 2014, 2, 19461-19472.

34 A. C. Baudouin, J. Devaux and C. Bailly, Polymer, 2010, 51, 1341-1354.

35 M. Sumita, K. Sakata, S. Asai, K. Miyasaka and H. Nakagawa, Polym. Bull., 1991, 25, 265-271.

36 D. K. Owens and R. Wendt, J. Appl. Polym. Sci., 1969, 13, 1741-1747.

37 R. Sondergaard, M. Helgesen, M. Jorgensen and F. C. Krebs, Adv. Energy Mater., 2011, 1, 68-71.

38 T. Stubhan, I. Litzov, N. Li, M. Salinas, M. Steidl, G. Sauer, K. Forberich, G. J. Matt, M. Halik and C. J. Brabec, J. Mater. Chem. A, 2013, 1, 6004-6009. 